\title{
Enhancing biogas production from agroindustrial waste pre-treated with filamentous fungi
}

\author{
Csilla Szücs $^{1}$ · Etelka Kovács ${ }^{1}$ - Zoltán Bagi ${ }^{1} \cdot$ Gábor Rákhely $^{1,2} \cdot$ Kornél L. Kovács $^{1,3}$
}

Received: 25 June 2020 / Accepted: 5 April 2021

๑) Akadémiai Kiadó Zrt. 2021

\begin{abstract}
Biogas is the product of anaerobic digestion (AD) of organic waste and is considered to be one of the most valuable natural renewable energy carriers. Plant biomass represents the most abundant eco-friendly energy reservoir on Earth. However, the tenacious and heterogeneous structure of the lignocellulose-rich elements makes it difficult for the involved microbes to digest the recalcitrant substrates. Both the degradation process and the biogas production yield can be enhanced by appropriate pretreatment of lignocellulosic materials. Filamentous fungi have been known as proficient colonizers of lignocellulosic plant tissues and have been recognized as producers of exceptionally rich and diverse hydrolytic enzymes. We tested Aspergillus nidulans, Trichoderma reesei, Rhizomucor miehei and Gilbertella persicaria filamentous fungal strains for pre-treatment of various agricultural lignocellulosic wastes. During the pre-treatment phase, the $\beta$-glucosidase and endoglucanase activity was measured spectrophotometrically. In the AD step, methane production was monitored by gas chromatography. The preliminary results showed that all the applied strains (Aspergillus nidulans, Trichoderma reesei, Rhizomucor miehei and Gilbertella persicaria) were highly effective in producing both $\beta$-glucosidase and endo-(1,4)- $\beta$-D-glucanase enzymes, which might explain the greatly improved $\mathrm{AD}$ results. Pre-treatment with the above-mentioned filamentous fungi positively affected the biogas production, although the effect strongly depended on the selection of the fungal partner for any given biomass substrate. Depending on the used substrate and the pre-treatment strain, overall methane yields were elevated two-fold relative to the controls.
\end{abstract}

Keywords Biogas $\cdot$ Biomass $\cdot$ Biomethane $\cdot$ Anaerobic digestion $\cdot$ Filamentous fungi $\cdot$ Hydrolytic enzymes

Csilla Szúcs

szucs.csilla@bio.u-szeged.hu

Etelka Kovács

kovacset@bio.u-szeged.hu

Zoltán Bagi

bagi.zoltan@bio.u-szeged.hu

Gábor Rákhely

rakhely@brc.hu

Kornél L. Kovács

kovacs.kornel@bio.u-szeged.hu

1 Department of Biotechnology, University of Szeged, Közép fasor 52, Szeged 6726, Hungary

2 Institute of Biophysics, Biological Research Centre, Temesvári krt. 62, Szeged 6726, Hungary

3 Department of Oral Biology and Experimental Dental Research, University of Szeged, Tisza Lajos körút 64-66, Szeged 6720, Hungary

\section{Introduction}

Our society's energy consumption tends to exceed fossil energy resources. Hence, one of the most relevant challenges for mankind today is to exploit the vast resources in the utilization of renewable energies. One of them is biogas, which is considered the most valuable among renewable energy carriers due to the environmental benefits associated with its production. Biogas is the product of anaerobic digestion (AD), a four-stage process including hydrolysis, acidogenesis, acetogenesis and methanogenesis. Various organic wastes can be degraded in AD and a carbon-neutral, renewable energy carrier (biogas) is generated (Lohri et al. 2014).

Plant biomass, a suitable substrate for AD, accumulates in massive amounts every year. Moreover, the food and feed industry and households produce tremendous amounts of organic waste on a daily basis that may fuel biogas production (Rogers et al. 2016). Biogas production from plant biomass is hampered by the poorly degradable lignocellulosic 
plant cell wall. The tenacious and heterogeneous structure makes the microbiological decomposition of lignocellulose difficult. Biogas production can be enhanced by appropriate pre-treatment of these materials, which facilitates the degradation process.

A process that is similar to $\mathrm{AD}$ also takes place in the digestive system of ruminants. Hay, forage and other nutrients, consumed by the animal are substrates for the microbial decomposition (Wirth et al. 2018). Ruminal decomposition of lignocellulose occurs in an anaerobic environment in the forestomach of the host animal. The microbial community structure is complex and diverse, the process involves several thousand bacterial strains (e.g. Clostridium, Fibrobacter, Ruminococcus, Ruminobacter) and methanogens (e.g. Methanobacterium, Methanobrevibacter, Methanomicrobium) (Agarwal et al. 2015).

Earlier studies have disclosed that the rumen usually contains filamentous fungi (De Almeida et al. 2012; Abrão et al. 2017). Filamentous fungi adapt to the rumen environment and colonize herbaceous components of the feed. Studies have proven that even the most lignified plant tissues can be colonized by fungi (Akin and Rigsby 1987). As producers of exceptionally rich and diverse hydrolytic enzymes, fungi contribute to the decomposition of cell wall polysaccharides (Jourdier et al. 2013; Boruta and Bizukojc 2017). This action is not only beneficial for the fungi but also helps the microbiome of the digestive system to further degrade the organic molecules.

Based on earlier reports (Abrão et al. 2017; Xiros et al. 2019), we isolated cellulose-degrading fungi from cattle rumen and employed them for pre-treatment of various lignocellulosic plant biomass sources to improve biogas production via facilitated degradation. Although several studies attempted pre-treatment of lignocellulose-rich substrates by microbes (e.g. Ahring et al. 1996; Taherzadeh and Karimi 2008; Alexandropoulou et al. 2017), this approach remained largely unexplored for AD.

\section{Materials and methods}

\section{Isolation and identification}

Filamentous fungus Aspergillus nidulans (BTT 002) was isolated from cattle rumen (E. Kovacs, unpublished). Rumen samples were obtained from abattoirs in Csongrád and Bács-Kiskun County, Hungary. Rumen contents were collected directly from six freshly slaughtered adult cows. The samples were transported in anaerobic containers and incubated at $37{ }^{\circ} \mathrm{C}$ before use for $1-2 \mathrm{~h}$. $20 \mu \mathrm{l}$ of each rumen sample were spread on carboxymethyl cellulose (CMC) plates, which contained $10 \mathrm{~g} \mathrm{CMC}$ (Merck, Darmstadt, Germany) and $15 \mathrm{~g}$ agar in $1000 \mathrm{ml}$ of distilled water. The medium contained $100 \mu \mathrm{g} / \mathrm{ml}$ of antibiotics (ampicillin, kanamycin, and streptomycin) to prevent bacterial infections. The plates were incubated at $37{ }^{\circ} \mathrm{C}$. After several re-plating on modified Czapek-Dox medium pure fungal cultures were obtained according to microscopic observation. The Czapek-Dox medium contained $2 \mathrm{~g} / \mathrm{l} \mathrm{NaNO}{ }_{3}, 1 \mathrm{~g} / \mathrm{K} \mathrm{K}_{2} \mathrm{HPO}_{4}, 0.5 \mathrm{~g} / 1 \mathrm{MgSO}_{4}, 0.5 \mathrm{~g} / \mathrm{l}$ $\mathrm{KCl}, 0.5 \mathrm{~g} / 1 \mathrm{FeSO}_{4}, 15 \mathrm{~g} / \mathrm{l}$ agar, $10 \mathrm{~g} / 1$ microcrystalline cellulose dissolved in $1,000 \mathrm{ml}$ of distilled water.

The ITS sequences of 12 pure isolates were amplified with polymerase chain reaction (PCR) using DreamTaq polymerase (ThermoFisher Scientific, Waltham, MA, USA) and the primer pairs ITS5-1737F/ITS2-2043R (Liu et al. 2017) and ITS3/ITS4 (Op De Beeck et al. 2014). The PCR cycling consisted of a first denaturation at $95^{\circ} \mathrm{C}$ for 2 min, followed by 32 cycles of denaturation at $95^{\circ} \mathrm{C}$ for $30 \mathrm{~s}$, annealing at $55^{\circ} \mathrm{C}$ for $30 \mathrm{~s}$ and elongation at $72{ }^{\circ} \mathrm{C}$ for $25 \mathrm{~s}$. The final extension was done at $72{ }^{\circ} \mathrm{C}$ for $5 \mathrm{~min}$.

Samples were sequenced at the Molecular Genomics Centre of the Biological Research Centre (Szeged) on a 3500 Series Genetic Analyzer (Life Technologies, Carlsbad, CA, USA).

The DNA sequences were filtered and analysed using BLAST against the NCBI database. Isolates with $\geq 99 \%$ sequence similarity to deposited sequences were considered identical species. A. fumigatus and A. nidulans were identified among the isolates. A. fumigatus, a known pathogenic strain, was eliminated.

\section{Biomasses used for pre-treatment}

Dry corn stover (CS), wheat straw (WS) and willow wood chip (WWC) samples were milled and sieved to a maximum particle size of $10 \mathrm{~mm}$ with an electric grinder (Retsch SM 100, Haan, Germany).

The total solid (TS) and organic total solid (oTS) contents of the substrates and the inoculum sludge (see later) were determined (Table 1). All measurements were performed in triplicates and the results are presented as mean values. The total solid (TS) content was measured after drying the biomass at $105^{\circ} \mathrm{C}$ overnight. The samples were then further heated at $550{ }^{\circ} \mathrm{C}$ for $1 \mathrm{~h}$ to obtain the organic total solid (oTS) fraction as recommended by the VDI 4630 protocol (VDI 4630, 2016).

Table 1 The total solid (TS) and organic total solid (oTS) contents of the substrates and the inoculum sludge. Values represent the mean of three parallel samples

\begin{tabular}{lcl}
\hline Substrate & Ts $(\%)$ & oTs $(\%)$ \\
\hline Sludge & 5.1 & 70.3 \\
WS & 9.5 & 96.1 \\
WWC & 15.4 & 97.3 \\
CS & 95.0 & 94.4 \\
\hline
\end{tabular}




\section{Pretreatment strains and samples}

Aspergillus nidulans was our own isolate as described above. Trichoderma reesei (BTT 001) strain was obtained from the strain collection of the Department of Biotechnology, University of Szeged (Hungary), while Rhizomucor miehei (SZMC 11,005) and Gilbertella persicaria (SZMC 11,086) strains were kindly provided from the Szeged Microbiological Collection (SZMC, Szeged, Hungary). Experiments were performed in triplicates and the results are given as mean values. Substrates were inoculated individually with each of these strains of filamentous fungi. The pre-treatment samples contained $1.46 \mathrm{~g}$ oTS lignocellulosic biomass. The sterilized substrates were added to $10^{7}$ conidia/sample in $20 \mathrm{ml}$ sterile distilled water. Samples were incubated at $37^{\circ} \mathrm{C}$ for 10 days.

\section{Enzyme activity assays}

Endo-(1,4)- $\beta$-D-glucanase activity was determined every third day during the pre-treatment process, using the 3,5-dinitrosalicylic acid (DNSA) method (Irfan et al. 2016). The absorbances were measured spectrophotometrically at $550 \mathrm{~nm}$ using a GENESYS UV-Visible scanning Spectrophotometer (ThermoFisher Scientific, Waltham, MA, USA). Calibration curve was established with DNSA (SigmaAldrich, ST. Louis, MO, USA). One unit of endoglucanase activity indicates the amount of enzyme required to release $1 \mu \mathrm{mol}$ glucose in $1 \mathrm{~min}$.

$\beta$-glucosidase activity was measured using $p$-nitrophenyl$\beta$-D-glucopyranoside ( $p$ NPG; (Sigma-Aldrich, ST. Louis, MO, USA) (Kaur et al. 2007). Absorbance readings were recorded at $400 \mathrm{~nm}$. Calibration curve was prepared using para-nitrophenol ( $p \mathrm{NP}$, Sigma-Aldrich, ST. Louis, MO, USA). One unit of $\beta$-glucosidase activity indicates the amount of enzyme required to release $1 \mu \mathrm{mol} p \mathrm{NP}$ in $15 \mathrm{~min}$.

Both enzyme activity measurements included sampling at day 0 (not shown on the diagrams). After every sampling, the supernatant liquid was discarded, and the reaction vessels were refilled with the same amount of sterile distilled water.

\section{Anaerobic digestion experiments}

Following pre-treatment, the samples were directly received $40 \mathrm{ml}$ of filtered sludge. This resulted in $60 \mathrm{ml}$ total volume of fermentation. The sludge came from a state-of-theart mesophilic biogas plant, which was fed by pig slurry and maize silage mix (Zöldforrás Biogas Plant, Szeged, Hungary).

Negative controls contained only sludge; the positive controls received sludge with microcrystalline cellulose as AD substrate. Samples containing non-pre-treated plant biomasses were also prepared to compare them with the pre-treated counterparts. Every AD digestion experiments were performed in triplicates and the results are given as mean values. AD reactors were incubated at mesophilic temperature for 25 days. Methane content of the headspace was measured daily via gas chromatography (Agilent $6890 \mathrm{~N}$ Gas Chromatograph, Agilent Technologies, Santa Clara, CA, USA).

\section{Statistical analysis}

Data of biomethane production were analysed by one-way analysis of variance (ANOVA, $p \leq 0,05$ ) and Duncan's test using the SigmaPlot 12 software.

\section{Results and discussion}

\section{Enzyme activity assays}

Endoglucanase enzymes are a group of hydrolases splitting the glucosilic bonds inside the cellulose polymer, which results in glucooligosaccharides (de Vries et al. 2001). Therefore, these enzymes play an important role in the degradation of lignocellulose. Endoglucanase enzyme activity results are summarized in Fig. 1. Activity values decreased in time for each biomass. This is likely due to the effect of decreasing degradable substrate availability or to the diminishing enzyme production in time. Overall, the highest specific activities were produced by A. nidulans on WWC and CS biomasses. In earlier studies, Aspergillus species were described as exceptional enzyme producers (de Vries et al. 2001; Abrão et al. 2017). The other three fungal strainsespecially $G$. persicaria -also presented high endoglucanase activity on WWC and CS substrates, respectively. Interestingly, WS biomass induced the lowest enzyme activity in the case of every fungal strain pre-treatment.

$\beta$-glucosidase is another key component of cellulolytic enzyme systems. This enzyme hydrolyses cellobiose by cleaving it into glucose molecules (Nutt 2006). $\beta$-glucosidase enzyme activity results are presented in Fig. 2 . The trends of the enzyme activity induction are somewhat similar to those observed with endoglucanase. A. nidulans showed good decomposition activities, especially when CS was the tested biomass. A. nidulans and G. persicaria also showed outstanding $\beta$-glucosidase production. WS triggered the smallest enzyme activity induction.

\section{Biomethane production}

A One-way Analysis of Variance (ANOVA) was conducted to compare the effects of filamentous fungal pre-treatment of various plant substrates (WWC, CS, WS) on biomethane 
Fig. 1 Endo-(1,4)- $\beta$-D glucanase enzyme activities of pre-treated substrates. (WWC , willow wood chip, $C S$, corn stover, $W S$, wheat straw). Values represent the mean of three parallel samples. The error bars indicate the deviation
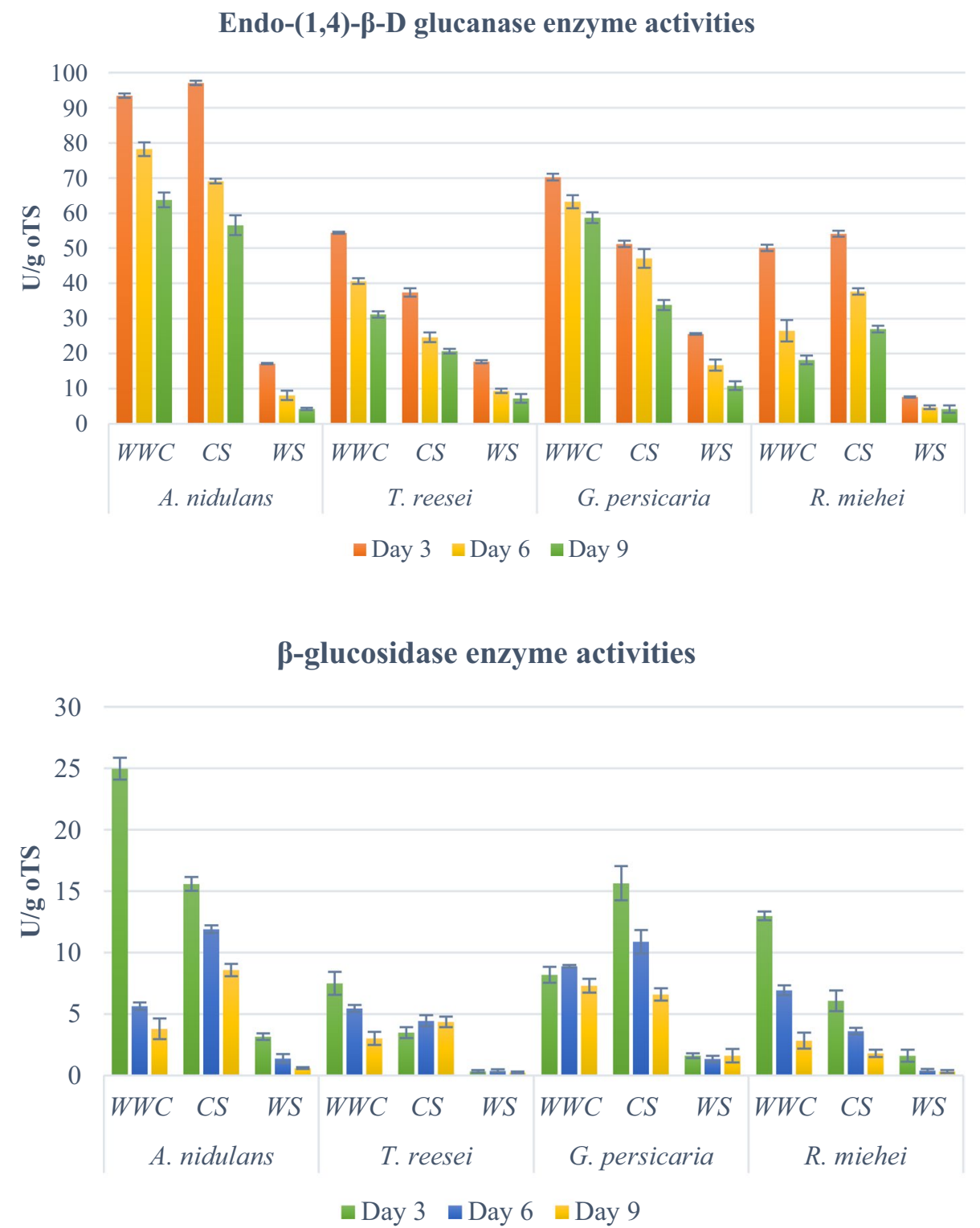

Endo-(1,4)- $\beta$-D glucanase enzyme activities

$\beta$-glucosidase enzyme activities

-Day 3 -Day 6 - Day 9
Fig. $2 \beta$-glucosidase enzyme activities of pre-treated substrates $(W W C$, willow wood chip, $C S$, corn stover, $W S$, wheat straw). Values represent the mean of three parallel samples. The error bars indicate the deviation production. There were significant differences at the $p<0.05$ level in case of WWC $[\mathrm{F}(4,10)=114.291, p<0.001]$.

Biomethane production and enzyme activity assay results correlated. Figure 3 shows that fungal pre-treatment increased methane yields in every combination relative to the control biomasses without pre-treatments CS biomass, pre-treated with A. nidulans, gave the highest methane yield, i.e. a substantial $84 \%$ increase relative to the positive control microcrystalline cellulose. The effect of the different strains was significant at $p<0.05$ level for the biomethane production regarding plant substrate CS $[\mathrm{F}(4$, $10)=86.302, p<0.001]$. When compared to the untreated CS $($ Mean $(M)=41.236$, Standard Deviation $(\mathrm{SD})=0.139)$, pre-treatment with $A$. nidulans $(M=84.388, \mathrm{SD}=3.063)$ doubled the methane yield $(p<0.001)$. A. nidulans pretreatment also differed significantly from pre-treatments by $T$. reesei $(M=66.191, \mathrm{SD}=4.310, p<0.001)$, G. persicaria $(\mathrm{M}=69.765, \mathrm{SD}=3.796, p<0.001)$, and $R$. miehei $(M=56.526, \mathrm{SD}=1.498, p<0.001)$, respectively.

Biomethane production was also notably distinct when WWC was applied as substrate. WWC samples pre-treated with $A$. nidulans $(M=57.252, \mathrm{SD}=3.506)$ differed significantly from samples pre-treated with $T$. reesei $(M=35.766$, $\mathrm{SD}=1.650, p<0.001), G$. persicaria $(\mathrm{M}=32.627$, $\mathrm{SD}=1.656, p<0.001), R$. miehei $(M=30.084, \mathrm{SD}=0.825$, $p<0.001)$ and from samples that have not received any kind of fungal pre-treatment $(M=27.202, \mathrm{SD}=0.626$, $p<0.001)$, respectively. Notwithstanding, samples pretreated with $G$. persicaria $(\mathrm{M}=32.627, \mathrm{SD}=1.656)$ were notably different from their corresponding controls $(\mathrm{M}=27.202, \mathrm{SD}=0.626, \mathrm{p}=0.008)$. No significant difference from either $T$. reesei $(M=35.766, \mathrm{SD}=1.650$, 
Fig. 3 Methane yields expressed as percentage of the positive controls. Different letters indicate statistically significant differences in sample groups, respectively ( $p \leq 0,05$, $n=3$ ). The results of methane production are expressed as percentage of the positive controls. Values represent the mean of three parallel samples. The error bars indicate the deviation

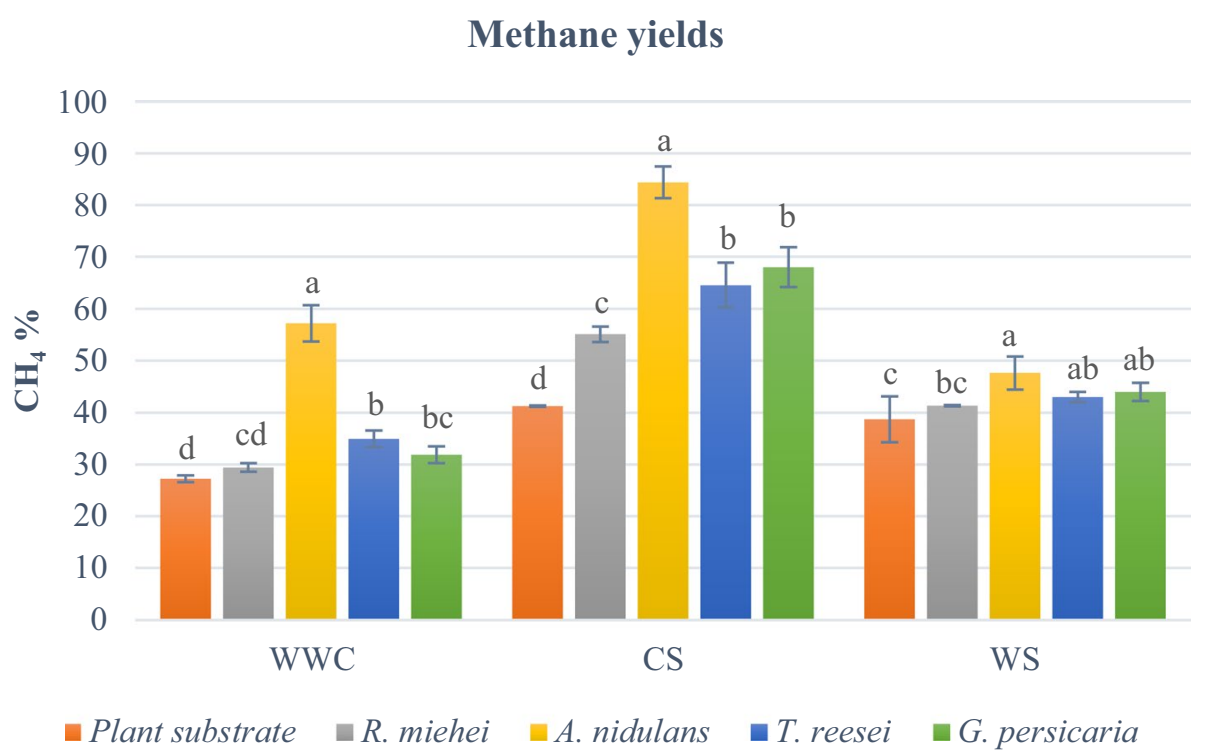

$p=0.076)$, nor $R$. miehei $(M=30.084, \mathrm{SD}=0.825$, $p=0.140)$ was detected.

Samples containing G. persicaria and T. reesei were not significantly different from each other $(p=0.174)$, although they differed significantly from samples pre-treated with $R$. miehei $(p<0.001$ and $p=0.003)$, and from "no pre-treatment" controls $(M=27.202, \mathrm{SD}=0.626, p<0.001$ in both cases).

In case of WS, significant effects of the different filamentous fungal strains were observed at $p<0.05$ level on the biomethane production $[\mathrm{F}(4,10)=4.928, p<0.001]$. Duncan's Multiple Range Test was used again as a comparison to examine the results. When WS was pre-treated with $A$. nidulans $(\mathrm{M}=47.668, \mathrm{SD}=3.197)$ results differed significantly from untreated fermentors $(M=38.703, \mathrm{SD}=4.402$, $p=0.003)$, and from samples that were pre-treated with R. miehei $(M=42.338, \mathrm{SD}=0.114, p=0.041)$. G. persicaria $(M=45.080, \mathrm{SD}=1.786)$ and $T$. reesei $(M=44.062$, $\mathrm{SD}=1.037)$ pre-treatments also resulted notable differences in biomethane production compared to samples containing only untreated plant substrates $(M=38.703, \mathrm{SD}=4.402$, $p=0.019$ and $p=0.036$ ), respectively. Nevertheless, samples pre-treated with $G$. persicaria and $T$. reesei did not indicate significant differences ( $p=0.643$ ).

This and all other results indicated that fungal pre-treatment facilitates subsequent decomposition of the biomass by the biogas forming microbial community. The results indicate a negative correlation between lignin content and methane yields. WWC is the most lignified substance among the tested substrates and consequently, it yielded the least biomethane production (except for the excellent performance by A. nidulans). Although filamentous fungi are able to colonize lignified plant materials, decomposition of lignocellulose remains an obstructive step during AD. Due to the high lignocellulosic content of WWC, longer or additional pre-treatment should be considered. Further experiments are necessary to optimize this step. In spite of the lower enzyme activity results, WS proved to be a better substrate in AD compared to WWC. The low TS and nutrient content of WS might have contributed to the moderate enzyme activity, which is in correlation with the methane yields of WS. CS proved to be the strongest element of our experiment. This substrate is characterized by elevated enzyme activity results and remarkable methane yields, which can be explained by the substrate's higher sugar and lower lignocellulose content. These properties and the material's structure promote the effortless microbial decomposition of CS. Overall, we can establish that the success of pre-treatment strongly depends on the fungal strain employed as well as on the biomass substrate.

\section{Conclusion for future biology}

This study demonstrated that the applied filamentous fungi proved to be excellent endo- $(1,4)-\beta$-D-glucanase and $\beta$-glucosidase sources for resourceful pre-treatment of lignocellulosic biomass. The $A$. nidulans isolate has shown outstandingly high endo- $(1,4)-\beta$-D-glucanase activity on WWC and CS, and high $\beta$-glucosidase activity on WWC. $G$. persicaria has also been shown to be an efficient endo$(1,4)-\beta$-D-glucanase producer especially on WWC and CS substrates. As a consequence of the pre-treatment, the biogas production in the samples pre-treated with A. nidulans was the highest for all raw materials involved. Pre-treatment with other fungal strains also improved decomposition efficiency and increased biogas yield in varying degrees. Based on these data, it is strongly recommended to use a short-e.g. 
10 days-fungal pre-treatment for cellulose-rich materials, which in certain cases can double the biogas yield.

All results support the conception, that the bioavailability of resistant plant wall biopolymers can be substantially improved. Attention should be paid, however, to the systematic and thorough selection of the appropriate filamentous fungus for the most effective deconstruction of the lignocellulosic biomass of various origin. In forthcoming utilizations properly designed biotechnological interventions may promote the exploitation of the lignocellulosic plant biomass for the efficient and economically attainable production of renewable energy in the form of biogas/biomethane. Adapting continuous biogas reactors to the pre-treated substrates is considered to be the next step in our way to industrial-scale application.

Acknowledgments We acknowledge the research funding received to carry out this study.

Author contributions $\mathrm{CsSz}$, with the help from EK and ZB, designed and performed the experiments and contributed to the evaluation of the data. KLK conceived the project and participated in its design. EK and KLK drafted the manuscript. GR critically evaluated the manuscript. All the authors have read and approved the final manuscript.

Funding This study has been supported in part by the Hungarian National Research, Development and Innovation Fund projects GINOP-2.2.1-15-2017-00081, GINOP-2.2.1-15-2017-00033, and EFOP-3.6.2-16-2017-00010. EK and ZB received support from the Hungarian NKFIH fund NKFI-PD 128345 and NKFIH FK123902, respectively. $\mathrm{CsSz}$ obtained a $\mathrm{PhD}$ fellowship from the University of Szeged.

\section{Declarations}

Conflict of interest The authors declare that the research was conducted in the absence of any commercial or financial relationships that could be construed as a potential conflict of interest.

\section{References}

Abrão FO, Duarte ER, Pessoa MS et al (2017) Notable fibrolytic enzyme production by Aspergillus spp. isolates from the gastrointestinal tract of beef cattle fed in lignified pastures. PLoS One. https://doi.org/10.1371/journal.pone.0183628

Agarwal N, Kamra DN, Chaudhary LC (2015) Rumen microbial ecosystem of domesticated ruminants. In: Rumen microbiology: from evolution to revolution. Springer India, pp 17-30

Ahring BK, Jensen K, Nielsen P et al (1996) Pretreatment of wheat straw and conversion of xylose and xylan to ethanol by thermophilic anaerobic bacteria. Bioresour Technol 58:107-113. https:// doi.org/10.1016/S0960-8524(96)00090-9

Akin DE, Rigsby LL (1987) Mixed fungal populations and lignocellulosic tissue degradation in the bovine rumen. Appl Environ Microbiol 53:1987-1995

Alexandropoulou M, Antonopoulou G, Fragkou E et al (2017) Fungal pretreatment of willow sawdust and its combination with alkaline treatment for enhancing biogas production. J Environ Manage 203:704-713. https://doi.org/10.1016/j.jenvman.2016.04.006

Boruta T, Bizukojc M (2017) Production of lovastatin and itaconic acid by Aspergillus terreus: a comparative perspective. World J Microbiol Biotechnol 33:1-12. https://doi.org/10.1007/ s11274-017-2206-9

De Almeida PNM, Duarte ER, Abrão FO et al (2012) Aerobic fungi in the rumen fluid from dairy cattle fed different sources of forage. Rev Bras Zootec 41:2336-2342. https://doi.org/10.1590/S151635982012001100006

de Vries RP, Visser J, Ronald P et al (2001) Aspergillus Enzymes involved in degradation of plant cell wall polysaccharides. Microbiol Mol Biol Rev 65:497-522. https://doi.org/10.1128/MMBR. 65.4.497

Irfan M, Asghar U, Nadeem M et al (2016) Statistical optimization of saccharification of alkali pretreated wheat straw for bioethanol production. Waste Biomass Valoriz 7:1389-1396. https://doi.org/ 10.1007/s12649-016-9540-2

Jourdier E, Cohen C, Poughon L et al (2013) Cellulase activity mapping of Trichoderma reesei cultivated in sugar mixtures under fed-batch conditions. Biotechnol Biofuels 6:1-12. https://doi.org/ 10.1186/1754-6834-6-79

Kaur J, Chadha BS, Kumar BA et al (2007) Purification and characterization of $\beta$-glucosidase from Melanocarpus sp. MTCC 3922. Electron J Biotechnol 10:260-270. https://doi.org/10.2225/vol10issue2-fulltext-4

Liu Z, Wang Y, Pan X et al (2017) Identification of fungal communities associated with the biodeterioration of waterlogged archeological wood in a Han Dynasty tomb in China. Front Microbiol 8:1-9. https://doi.org/10.3389/fmicb.2017.01633

Lohri CR, Diener S, Zurbrügg C (2014) Anaerobic digestion of biowaste in developing countries-practical information and case studies VUNA-nutrient recovery from urine view project resource recovery and reuse view project

Nutt A (2006) Hydrolytic and oxidative mechanisms involved in cellulose degradation. Digit Compr Summ Uppsala Diss from Fac Sci Technol NV - 185:51

Op De Beeck M, Lievens B, Busschaert P et al (2014) Comparison and validation of some ITS primer pairs useful for fungal metabarcoding studies. PLoS ONE. https://doi.org/10.1371/journal. pone.0097629

Rogers JN, Stokes B, Dunn J et al (2016) An assessment of the potential products and economic and environmental impacts resulting from a billion ton bioeconomy. Biofuels, Bioprod Biorefining. https://doi.org/10.1002/bbb.1728

Taherzadeh MJ, Karimi K (2008) Pretreatment of lignocellulosic wastes to improve ethanol and biogas production: A review. Int J Mol Sci 9:1621-1651. https://doi.org/10.3390/ijms9091621

VDI 4630 (2016) Fermentation of organic materials - Characterization of the substrate, sampling, collection of material data, fermentation tests. VDI- Handbuch Energietechnik. Deutsches Institut fur Normung E.V. (DIN), Düsseldorf

Wirth R, Kádár G, Kakuk B et al (2018) The planktonic core microbiome and core functions in the cattle rumen by next generation sequencing. Front Microbiol 9:2285. https://doi.org/10.3389/ fmicb.2018.02285

Xiros C, Shahab RL, Studer MHP (2019) A cellulolytic fungal biofilm enhances the consolidated bioconversion of cellulose to short chain fatty acids by the rumen microbiome. Appl Microbiol Biotechnol 103:3355-3365. https://doi.org/10.1007/ s00253-019-09706-1 